\title{
Risk management for bridges: a case study of unforeseen failure mode
}

\author{
Alessandro Pucci, Hélder S. Sousa, José C. Matos
}

ISISE, Institute of Science and Innovation for Bio-Sustainability (IB-S), Department of Civil Engineering, University of Minho, Guimarães, PT

\section{Mario Lucio Puppio, Linda Giresini}

Department of Energy, Systems, Territory and Constructions Engineering, University of Pisa, Pisa, IT

\author{
Mauro Sassu \\ Department of Civil and Environmental Engineering and Architecture, University of Cagliari, \\ Cagliari, IT
}

Contact: ale@civil.uminho.pt

\begin{abstract}
Risk management plays a crucial role in the stakeholders' decision making because it is directly related to safety, serviceability and economy. There is now a growing concern about how to relocate known risks into an acceptance threshold: this implies the evaluation of several options obtained from hazard scenarios considering the related consequences. In parallel, practitioners usually rely on standard tools for risk assessment, and on structural codes to compute performances. Although this approach is currently widely implemented, this research shows that hazardous situations can arise in properly designed infrastructures, due to errors in management. This paper deals with such issue, also highlighting a gap in current codes that could contribute to losses caused by unforeseen failure modes. In this study, a preliminary FMEA assessment was performed to identify the failure modes that required a deeper quantitative analysis. In a second step, a quantitative analysis was implemented, using a modular methodology that combines reliability theory with a risk-based approach. The results evidenced that a wider analysis focused on the identification of vulnerable areas shall be considered in every stage of the asset management. Furthermore, the dynamic of this process is regulated by the established safety level concerning possible damages to people, production sites and commercial activities.
\end{abstract}

Keywords: Risk Analysis, Decision-making, Reliability Theory, Bridge failure, Flood Hazard, Hydrology, Bridge Management System, Probability, Infrastructure Management, Buoyancy.

\section{Introduction}

The management of the built environment is one of the most relevant present and future challenge for engineers. In particular, the safety and functionality of bridges plays a key-role on the mobility of people and goods. In this scenario, each bridge failure has direct and indirect economic consequences that might be relevant at both local and country scale [1]. Hence, is not 
surprising that the scientific community revealed a growing interest in the analysis of past collapses. Lessons learned by these investigations, represent not only a pedagogic tool, but also the basis for future improvements of codes and standards [2]. A recent literature review on failures' statistics confirmed that hydraulic-related failures dominates both in the overall mean and in the number of collapses per single event [2]. The concern, from a designer point of view, relies on current codes of practice. Hydraulic actions are not often properly accounted in standards, even though the worldwide scenario is rather fragmented. Several countries as USA and Australia specifically account for water actions on bridges, while the Eurocodes lack of an exhaustive formulation. In fact, the EN 1997 [3] provides some Limit States but mostly referred to geotechnical aspects, while hydraulic loads are considered only during construction. Although EN 1991-1-6 gives few indications, the main issue is that it covers only the building phase, leaving to the designer the burden of establishing a proper hydraulic model and define some plausible limit states for the whole lifespan of the bridge. This is mainly related to buoyancy and dragging limit states, while the geotechnical assessment is better covered. Hence, at least in Europe, there is the need to properly address water actions on bridges. The scientific community developed reliability tools for the assessment of new Limit States (LS) to account for a failure mode that is not currently covered by standards. Reliability analysis is usually applied to measure structural performances. This because reliability theory underpins current codes and is used for the calibrations of safety levels [4]. Despite the existence of such advanced methods, there is a considerable gap between practitioners and academia [5]. Furthermore, the decision-making process is inevitably linked to economic aspects that cannot be explained by means of a reliability analysis [6]. Instead, risk indices are by definition performance indicators suitable to account for the economic consequences of a certain loss. From these considerations it is clear that in the light of future managements, reliability and risk analysis should always be coupled, to ensure a balance between safety levels and associated costs. The paper presents a framework that combines a reliability-based module with a risk-based approach. The methodology is illustrated in Section 2 and applied to a case-study in Section 3.

\section{Methodology}

The proposed framework is composed by two modules; the first is a reliability analysis applied to the hydraulic uplift action on a bridge deck. It uses the results obtained from visual inspections and a hazard simulation analysis. The second module involves the mitigation actions needed to reduce the risk, if is deemed unacceptable. The risk index is obtained in a decision-making context, evaluating each alternative based on its costs and time needed to be realized. The method is illustrated in Figure 1.

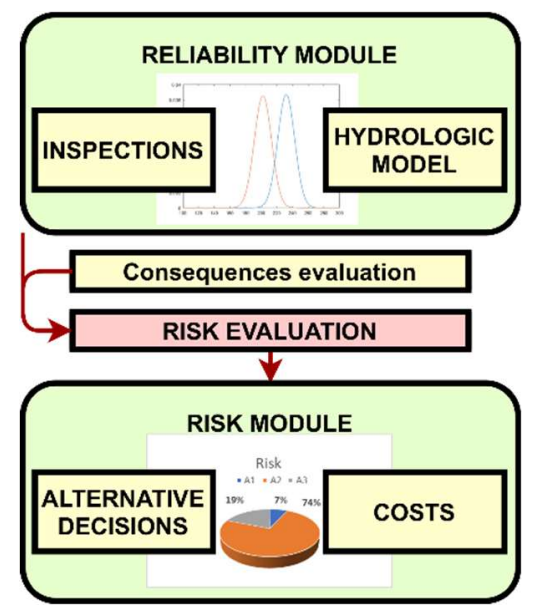

Figure 1. Flowchart of the method

\subsection{Reliability Module}

The safety level of the asset is evaluated using the methodology presented in Figure 2. Firstly, a visual inspection is carried out to define the system's characteristics, according for example to the methodology proposed by Pucci et al., (2019) [7]. Then, a Failure Mode and Effect Analysis (FMEA) is carried out to identify the possible failures of the system components and subcomponents. The behaviour of each element is qualitatively investigated regarding the same hazard. Then, a quantitative analysis is used for the most critical component according to its consequences in case of failure. 


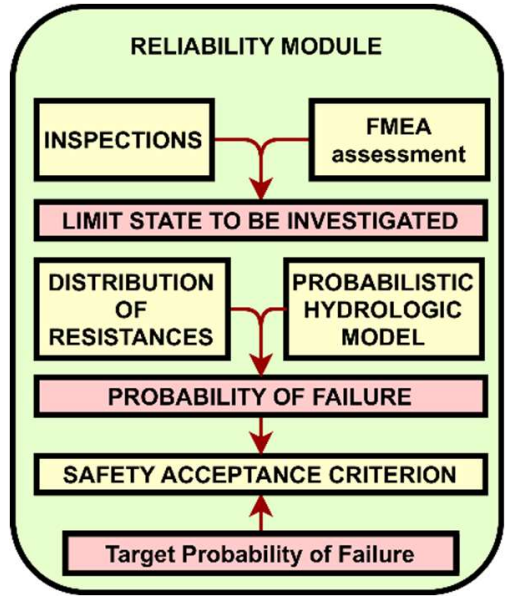

Figure 2. Reliability module flowchart

In this study, the buoyant action is characterized by equation (1).

$$
\mathrm{G}=\mathrm{R}-\mathrm{S}=\mathrm{W}_{\text {deck }}-\mathrm{P}_{w}
$$

Where, $W_{\text {deck }}$ is the deck self-weight, and $P_{w}$ is the hydrostatic uplift thrust against the deck. The stabilizing action, denoted with $\mathrm{R}$, is computed using the geometric data obtained through the inspections. The variability of the data represents the source of uncertainty and is promptly considered. On the load side, the uplift action is evaluated using equation (2).

$$
\mathrm{P}_{w}=\gamma_{w} \cdot b \cdot a \cdot x
$$

Where, $\gamma_{w}$ is the water density, $b, a$ and $x$ are represented in Figure 3. In particular, $x$ is the free board of the water flow set zero at the bridge's intrados. On the action' side the only probabilistic parameter is the river discharge $(Q)$ that can be computed using an appropriate hydraulic model [8]. In the proposed analysis, for each return period of the action $\left(T_{R}\right)$, the variability of $Q$ is accounted for. This means that the hydraulic model is computed $\forall t_{R} \in T_{R}=\{2,3, \ldots, 200$ years $\}$. The computational burden results in 199 simulations, and for each $T_{R}$ a maximization and a minimization of random parameters is carried out to generate the distribution of $Q\left(T_{R}\right)$. A significant increment of cost-effectiveness is obtained by reducing the number of simulations from 199 to 5 by means of a logarithmic interpolation performed in the chart $Q=Q\left(T_{R}\right)$. The lowest and the highest return periods should be simulated in order to do not compute extrapolations. The beta index can be then evaluated for each $T_{R}$ using the First Order Reliability Method (FORM). This allows to probabilistically quantify the buoyancy of the bridge deck in the occurrences in which this analysis is relevant, i.e. supported girders. Although the analysis can be also performed by simplified methods [9], the buoyancy action is very sensitive to the bridge's boundary conditions. In particular, the height of the riverbanks, the shape of the girder and the presence of new bridges might change dramatically the safety level. This issue is relevant as an original safe design against the buoyancy LS might become unsafe due to hydraulic works operated downstream or upstream the bridge. In such perspective, the manager should always perform a check through hands-on tools if the planned works are causing changes on safety levels within the portfolio of structures. The final step of this module is to check whether the reliability indices are deemed acceptable or not. The comparation is obtained using target reliability indicators provided in literature [10].

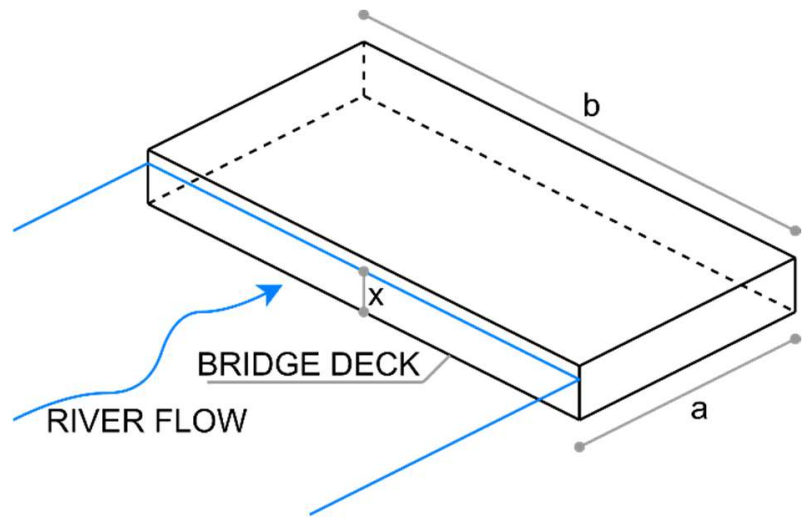

Figure 3. Scheme of the deck partially submerged

\subsection{Risk Module}

In the management of a bridge, it might happen at a certain point in the lifespan of the structure, that the reliability target for a certain LS cannot be met. In such scenario, the causes could be determined by many factors like design, construction error, or a management error; this paper focuses on the latter, highlighting the role of an unforeseen failure mode (i.e. buoyancy) caused by works alongside the river, as new embankments, bridges or dams. The 
consequences on economy, society and environment due to a bridge failure can be assessed using a risk-based approach. Risk, and more specifically flood risk, is analysed through different formulations depending on the context to which is applied (e.g. social, technical, economic, environmental). A review on such metrics can be found in [11]. The common features of all definitions are: (i) the assessment of a probability and (ii) the loss estimation in case the event occurs. A rational approach in flood risk assessment is the so-called Source-PathwayReceptor framework [12]. Uncertainties propagates from the raw hydrometric data (source) to the civil infrastructures (receptors) through the watercourses (pathways). In the proposed framework, the reliability module already considers this chain of events. Hence, what is still missing is the consequences evaluation. This can be appraised if the considered area already suffered from inundations. An estimate of damages can be obtained from past events by linking the spatial pathway of the flooded area to the return period of the event. Then, a qualitative risk scale, e.g. Hicks scale, can be adopted for the preliminary evaluation of flood risk regarding the selected LS; i.e. buoyancy. If the risk is deemed acceptable, interventions should be performed to reduce either the probability of occurrence or the consequences. In case of buoyancy LS, several actions can be implemented using structural and non-structural countermeasures. After the evaluation of the costs associated to each alternative solution (e.g. new bridge or lowering the riverbed among others), the decision should be based also on criteria as the time needed to complete the works, the long-term costs of the decision alternative, the sensitivity of the manager to losses. Approaches of these criteria to the utility and cumulative prospective theories can be found in [13]. In the present work, the accent is stressed on the importance of urgent measures to mitigate the risk in case the safety requirements are not met for rather low hazard return periods $1<100$ years). In this scenario, during the flood event, it is crucial to implement non-structural measures. An early warning system can be employed through the combination of a real-time monitoring of the river's discharge and the use of the hydrograph obtained during the hazard simulations. Given the hydrograph in Figure 4, three discharges can be identified: $Q_{c}$ is the flow that corresponds to the beginning of the inundation, $\mathrm{Q}_{b}$ is the discharge corresponding to the $\beta_{\text {target, }}$ i.e. the reliability threshold for the computed LS. Finally, $Q_{A}$ is related to the time $t_{A}$ in which the Civil Protection alert should be raised. Hence, the minimum time needed to implement measures to mitigate the consequences of a flood is $t_{c}-t_{b}$. This early warning system is a temporary measure in case the risk level is deemed unacceptable and risk-control actions should be promptly taken. In such occurrence, the early warning cost should be accounted in the planned decision alternative. The risk associated to different options can be evaluated assuming as probability of occurrence the formula given in equation 3 .

$$
\mathrm{P}_{i}=1-\left(1-\frac{1}{T_{R}\left(Q_{c}\right)}\right)^{N_{i}}
$$

Where, $\mathrm{N}_{\mathrm{i}}$ is the time (in years) needed to complete the construction works related to the ith decision alternative. The decision-related risk is assessed according to equation 4.

$$
\mathrm{r}_{\mathrm{i}}=P_{i} \cdot\left(€_{i}+€_{E W}\right)
$$

Where, $€_{\mathrm{EW}}$ is the total cost of the early warning system for the planned time $\mathrm{N}_{\mathrm{i}}$, and $€_{\mathrm{I}}$ is the direct cost of the $\mathrm{i}$-th decision. A raking of all alternatives allows to determine the most convenient one in terms of construction costs, probability of exceedance of the selected LS during the works, and early warning system costs. The overall framework is demonstrated in the next section on a real case-study located in Tuscany (Italy).

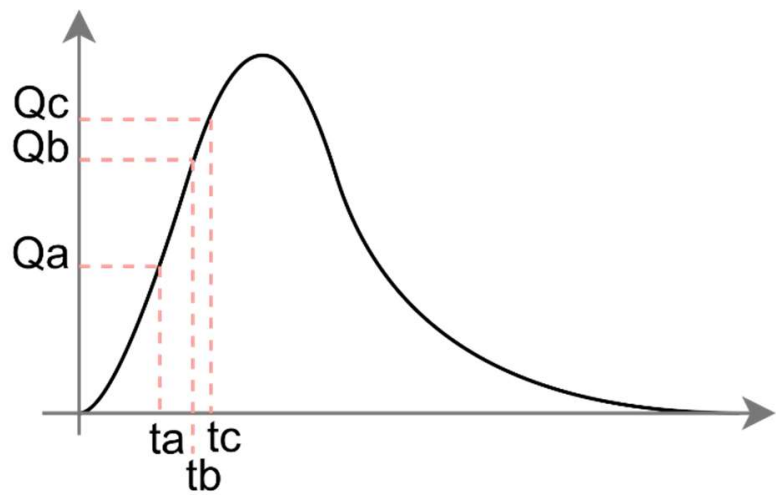

Figure 4. Hydrograph to aid early warning systems 


\section{Application}

The method is applied to a bridge located in via Menconi - via Giovan Pietro in Avenza, Italy. The bridge, presented in Figure 5 was built in 2007. The planned works involved the demolition and reconstruction of two bridges: the one in via Menconi and another 400m downstream located in via Covetta. The management of the two structures is held by the same public institution.

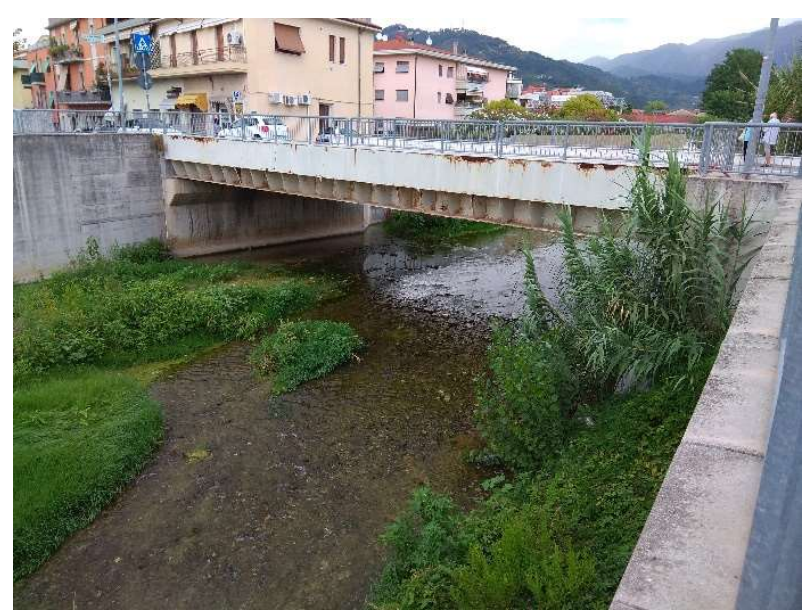

Figure 5. Via Menconi - Via Giovan Pietro Bridge

\subsection{Reliability module}

An inspection campaign was performed on 2018 and did not highlighted defects nor severe aging of the bridge [7]. The FMEA analysis, revealed that a possible buoyancy and dragging of the deck is not restrained, as the bearings only have $15 \mathrm{~mm}$ shear studs against lateral actions. Hence, if the uplift thrust during a flood is great enough to lift the deck more than $15 \mathrm{~mm}$, then the deck can be washed away. A possible resulting failure in the chain of events is the obstruction of the water flow by the dragged deck. This will lead to the overtopping of embankments and a generalised inundation of the surrounding city, which is built in the river's floodplain.

\subsubsection{Probabilistic characterization of the inputs}

The bridge deck, being not restrained for uplift actions, contrasts the Archimede's thrust only through its self-weight. The distribution of the deck weight is based on the information obtained during the inspection campaign. On the contrary, the river's discharge is probabilistically characterized through a distributed hydrologic model as the one developed by Castelli et al., 2014 [8]. The model uncertainty is probabilistically considered and summarized in Table 1.

Table 1. Parameters of the distributions

\begin{tabular}{cccc}
\hline $\begin{array}{c}\text { Action } \\
{[-]}\end{array}$ & $\begin{array}{c}\text { Mean } \\
{[-]}\end{array}$ & $\begin{array}{c}\text { St. Dev. } \\
{[-]}\end{array}$ & $\begin{array}{c}T_{R} \text { (for Q) } \\
\text { [years] }\end{array}$ \\
\hline$W_{\text {deck }}[$ ton] & 231,9 & 10,8 & - \\
\hline$Q\left[\mathrm{~m}^{3} / \mathrm{s}\right]$ & 100,3 & 2,5 & 2 \\
\hline$Q\left[\mathrm{~m}^{3} / \mathrm{s}\right]$ & 302,5 & 3,0 & 30 \\
\hline $\mathrm{Q}\left[\mathrm{m}^{3} / \mathrm{s}\right]$ & 340,7 & 3,2 & 50 \\
\hline $\mathrm{Q}\left[\mathrm{m}^{3} / \mathrm{s}\right]$ & 392,4 & 3,3 & 100 \\
\hline $\mathrm{Q}\left[\mathrm{m}^{3} / \mathrm{s}\right]$ & 444,2 & 3,5 & 200 \\
\hline
\end{tabular}

Before computing the reliability analysis, a proper hydraulic study should be performed, and is illustrated in the next section.

\subsubsection{Hydraulic modelling and buoyancy effect}

The modelling of the open-channel hydraulic is done using De Saint Venant equations, implemented in a commercial software. The analysed stretch of the watercourses goes $500 \mathrm{~m}$ upstream the bridge until $1000 \mathrm{~m}$ downstream it. The upper and lower limits were selected considering the presence of other hydraulic manufacts in the same river (e.g. the via Covetta bridge, located $400 \mathrm{~m}$ downstream the analysed structure). This analysis, that in the specific casestudy was aided using the software HEC-RAS [14], reported an anomalous behaviour of the river flow, downstream the analysed bridge. It was observed a significant backwater effect due to the contemporary presence of the via Covetta bridge and a narrowing of the river's cross section. This caused a significant increase on the free board of the water flow. The comparison in the absence and in presence of such obstacles is highlighted in Figure 6 . It can be clearly seen that the backwater dramatically increases the uplift thrust just after the water's free board reaches the via Menconi bridge intrados. The analysis is computed for $T_{R}$ ranging between 2 and 30 years, given an already buoyant deck at $T_{R}=30$ years. 


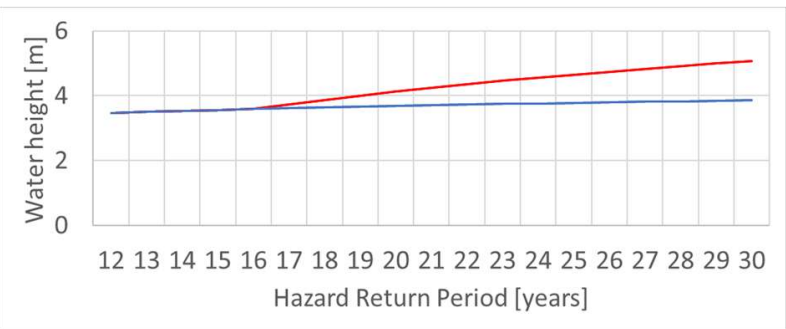

Figure 6. Water height value for different $T_{R}$

Thus, the bridge is exposed to an unforeseen limit state that should have been accounted during the rebuilding phase in 2007. The design choices in the via Covetta bridge aimed to respect the river's width, maintaining the original $16 \mathrm{~m}$, while in the bridge of via Menconi, the cross section widen to $19 \mathrm{~m}$, given the impossibility to increase the bridge clearance due to urbanistic constraints. Hence, the new structure costed 0.7 million Euro, although designed according to the existing codes, is not safe regarding the buoyancy of its deck.

\subsubsection{Reliability Index of buoyancy Limit State}

The probability of failure regarding the buoyancy Limit State, that is a global failure, is shown in Table 2. The results are compared to the target $\beta$ index, that is chosen equal to 3.8 as a minimum safety level; it is indeed an unusual value for a bridge, since the design $T_{R}$ is usually 100 years. The critical condition is reached for $T_{R}=25$ years, as the definitive proof of inadequacy against uplift actions.

Table 2. Reliability index for buoyancy $L S$

\begin{tabular}{ccc}
\hline $\begin{array}{c}T_{\mathbf{R}} \\
\text { [years] }\end{array}$ & $\begin{array}{c}\text { Probability of failure } \\
{[-]}\end{array}$ & $\begin{array}{c}\text { Beta } \\
{[-]}\end{array}$ \\
\hline 23 & $6,7 * 10^{-7}$ & 4,83 \\
\hline 24 & $6,6 * 10^{-5}$ & 3,8 \\
\hline 25 & $4,0 * 10^{-3}$ & 2,63 \\
\hline
\end{tabular}

\subsection{Risk module}

The consequences of an inundation are estimated from historical data, as many floods occurred in the same area during the last two decades. The closest one concerning the outbreak point of the river resulted in 24 million Euro damages [15].
Thus, applying the Hicks scale, the risk is deemed unacceptable (Weight $=3$, Consequences $=60$; Risk $=3 * 60>100)$ and risk-mitigation measures should be taken. The associated costs were estimated using analogous works already planned or executed on the same river. The time needed is estimated using real Gantt diagrams. The early warning system is considered to be the same for all the three alternative decisions; hence, in this particular case, it is not included in the costs computation. From the analysis of the hydrograph for $T_{R}=30$ years in Figure 7, has been found that the minimum time to issue a short-notice warning is 15 minutes.

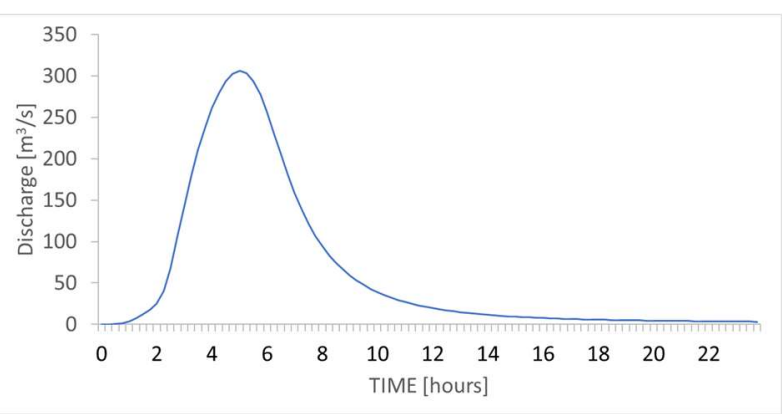

Figure 7. Hydrograph for $T_{R}=30$ years

\subsection{Results and discussion}

The analysis revealed that a generalised inundation might occur for rather low return period of the hazard ( 30 years). Although the bridge was designed according to current standards, changes in the river's geometry led to unexpected heighten of the water profile. This is the cause of the cited backwater effect. The performed reliability assessment was coupled to a qualitative risk acceptance criterion to decide whether actions are needed. Three options were found: (A1) demolition and rebuilding of the via Menconi bridge; (A2) demolition and rebuilding of the embankments downstream the bridge and widening of the river's cross section; (A3) lowering of the riverbed, using a small trapezoidal section to limit the impact of such measure on the embankments. The costs associated to these options are: (A1) 0,56 million Euro; (A2) 2,1 million Euro; (A3) 1,2 million Euro. The Gantt diagrams led to the following durations: (A1) 6 months; (A2) 1,5 years; (A3) 8 months. The latter is a less local intervention because is planned until the river's outlet section; this justify the time needed for its 
completion. The resulting risk pie chart is shown in Figure 8.

\section{Risk}

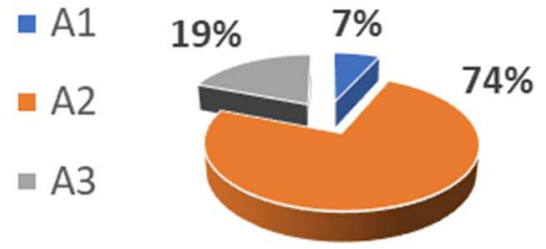

Figure 8. Risk associated to the three alternatives

Hence, it can be concluded that the best alternative is A1. Other criteria based on utility or cumulative prospective theory shall be further included. This will allow to search for the optimal risk-informed decision. In addition, indirect costs related to economic losses of commercial activities shall be included if option A1 is chosen, while A3 does not have such additional costs. Regarding the hazard simulation, the logarithmic interpolation performed on $Q\left(T_{R}\right)$ to reduce the number of simulations, not only improved the computational efficiency of the method, but revealed also a good coefficient of determination $\left(R^{2}=0.99\right)$, as displayed in Figure 9.

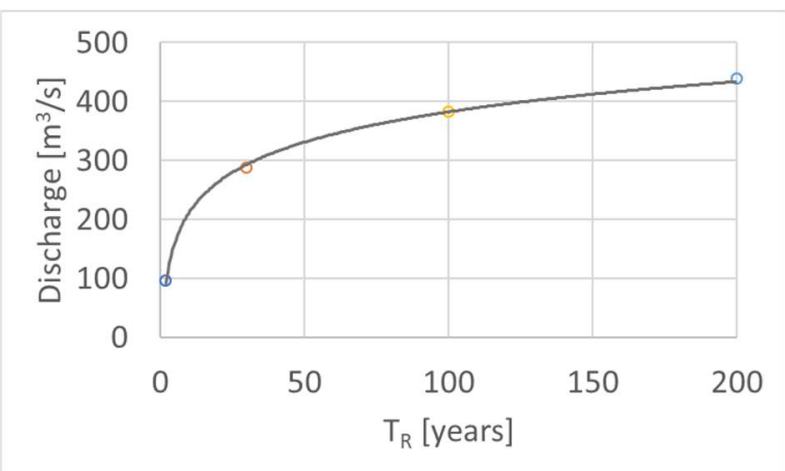

Figure 9. Discharge interpolation for different $T_{R}$

\section{Conclusions}

The developed framework pointed out the need of better infrastructures management procedures, using tools as reliability and risk analysis. The preliminary qualitative analysis of failure modes highlighted the most relevant ones in relation to their expected consequences; the use of reliability analysis was decisive to quantify the severity of the failure in relation to the hazard return period. However, this has been made possible only because a local hydraulic analysis was also performed. Indeed, the responsible of the buoyancy was found to be the backwater effect. This was highlighted only from the simulations performed in HEC-RAS. The unforeseen failure mode was proven to be determinant for very low return periods (around 25 years); the analysis of expected consequences allowed to quantify the risk. Acceptance criteria were employed to decide the typology of interventions to be taken. Furthermore, the hydrograph used in the simulations was also used to quantify the early warning notice in terms of available time to evacuate the population from the floodable areas. Further investigations on decision criteria will help to optimize the choices helping the bridge manager.

\section{Acknowledgements}

This work was partly financed by FEDER funds through the Competitivity Factors Operational Programme - COMPETE and by national funds through FCT Foundation for Science and Technology within the scope of the project $\mathrm{POCl}-$ 01-0145-FEDER-007633. This work was supported by the FCT Foundation for Science and Technology under Grant SFRH/BD/145478/2019. This project has received funding from the European Union's Horizon 2020 research and innovation programme under grant agreement No 769255 . This document reflects only the views of the author(s). Neither the Innovation and Networks Executive Agency (INEA) nor the European Commission is in any way responsible for any use that may be made of the information it contains.

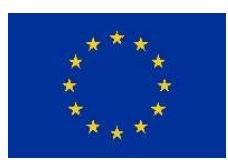

\section{References}

[1] Wang W, Stanley HE, Gao J. Local floods induce large-scale abrupt failures of road networks. Nat Commun [Internet]. Springer US; 2019;10:1-11. Available from: http://dx.doi.org/10.1038/s41467019-10063-w

[2] Proske D. Bridge Collapse Frequencies versus Failure Probabilities. Risk Engin. Cham: Springer 
International Publishing; 2018.

[3] CEN. Eurocode 1: Actions on Structures [Internet]. Brussels: European Committee for Standardization; 2004. Available from: https://eurocodes.jrc.ec.europa.eu/

[4] Ghosn M, Duenas-Osorio L, Frangopol DM, McAllister $M$, Bocchini $P$, Manuel $L$, et al. Performance Indicators for Structural Systems and Infrastructure Networks. J Struct Eng. 2016;142:F4016003.

[5] Biondini F, Frangopol DM. Life-Cycle Performance of Civil Structure and Infrastructure Systems : Survey. J Struct Eng. 2018;144:06017008.

[6] Akiyama M, Frangopol DM, Ishibashi H. Toward life-cycle reliability-, risk-, and resilience-based design and assessment of bridges and bridge networks under independent and interacting hazards: emphasis on earthquake, tsunami and corrosion. Struct Infrastruct Eng [Internet]. Taylor \& Francis; 2020;16:26-50. Available from: https://doi.org/10.1080/15732479.2019.1604770

[7] Pucci A, Sousa HS, Puppio ML, Giresini L, Matos JC, Sassu M. Method for sustainable large-scale bridge survey.pdf. Towar a Resilient Built Environ Risk Asset Manag. Zurich: IABSE; 2019. p. 103441.

[8] Yang J, Castelli F, Chen Y. Multiobjective sensitivity analysis and optimization of a distributed hydrologic model MOBIDIC. Hydrol Earth Syst Sci Discuss. 2014;11:3505-39.

[9] Puppio ML, Novelli S, Sassu M. Failure evidences of reduced span bridges in case of extreme rainfalls the case of Livorno. Frat ed Integrita Strutt. 2018;12:190-202.

[10] Sykora M, Holicky M, Lenner R, Manas P. Target Reliability Levels for Existing Bridges Considering Emergency and Crisis Situations. Adv Mil Technol. 2014;9:45-57.

[11] Vorogushyn S, Bates PD, De Bruijn K, Castellarin A, Kreibich $\mathrm{H}$, Priest $\mathrm{S}$, et al. Evolutionary leap in large-scale flood risk assessment needed. Wiley Interdiscip Rev Water. 2018;5:1-7.

[12] Hall J. Modelling for Flood Risk Management.
In: Christie M, Cliffe A, Dawid P, Senn S, editors. Simplicity, Complex Model. Statistics. Chichester: John Wiley \& Sons, Ltd; 2011. p. 125-46.

[13] Gong C, Frangopol DM. Condition-Based Multiobjective Maintenance Decision Making for Highway Bridges Considering Risk Perceptions. J Struct Eng. 2020;146:040200051.

[14] USACE. HEC-RAS [Internet]. 2020 [cited 2020 May 26]. Available from: https://www.hec.usace.army.mil/software/hecras/

[15] Pucci A. Bridge Network Failure due to extreme rainstorms. University of Pisa; 2019. 